\title{
SET-CAN Fusion Gene in Acute Leukemia and Myeloid Neoplasms: Report of Three Cases and a Literature Review
}

This article was published in the following Dove Press journal: OncoTargets and Therapy

\author{
Heyang Zhang' \\ Lijun Zhang' \\ Yan $\mathrm{Li}^{\mathrm{I}}$ \\ Hongcang $\mathrm{Gu} \mathbb{D}^{2}$ \\ Xiaoxue Wang $\mathbb{D}^{1}$ \\ 'Department of Hematology, The First \\ Hospital of China Medical University, \\ Shenyang, Liaoning, People's Republic of \\ China; ${ }^{2}$ Broad Institute of MIT and \\ Harvard, Cambridge, MA, USA
}

Objective: To investigate the characteristics of hematological malignancies in patients with the SET-CAN fusion gene and provide a literature review.

Methods: We retrospectively analyzed the clinical data of three cases of acute leukemia and myeloid neoplasms harboring the SET-CAN fusion gene who were treated at our hospital. Their clinical manifestations, pathological results and treatment strategies were investigated. Results: The three cases were diagnosed with T-cell acute lymphoblastic leukemia (T-ALL), acute myeloid leukemia (AML) and myeloid sarcoma (MS), respectively. Karyotype analyses identified a normal result in all three patients. Subsequently, we confirmed del(9q34) utilizing FISH analysis. Mutation of the $B R A F$ gene was detected in case 1, while mutations in PHF6 and BCOR were detected in case 2, which have not been officially reported in patients with SET-CAN fusions. Finally, relevant literature focusing on adult patients with hematological malignancies harboring the SET-CAN fusion gene were summarized.

Conclusion: Adult patients with the SET-CAN fusion gene were rare among cases of hematological malignancies. There was a large degree of heterogeneity between different patients. Notably, some patients remained sensitive to chemotherapy. Overall prognosis may be related to the type of disease and other cytogenetic abnormalities. Systemic cytogenetic and molecular studies are needed to make accurate diagnoses. Additional cases need to be accumulated and summarized to better understand these diseases.

Keywords: T-lymphoblastic lymphoma/leukemia, acute myeloid leukemia, myeloid sarcoma, SET-CAN, ASCT, prognosis

\section{Introduction}

Recurrent genetic abnormalities are considered to be diagnostic and prognostic markers in patients with hematological malignancies. ${ }^{1,2}$ Although intensive chemotherapy and allogeneic stem cell transplantation have greatly contributed to therapeutic strategies, it is still difficult to guarantee long survival and predict clinical outcomes for many individuals. More studies focusing on cytogenetic aberrations and molecular abnormalities are required for further exploration. ${ }^{3}$ The SET-CAN/NUP214 fusion gene is a relatively rare genetic event in leukemia. It was first detected in a patient with acute undifferentiated leukemia (AUL) ${ }^{4}$ and was later detected in a patient with acute myeloid leukemia (AML). ${ }^{5}$ Subsequently, additional T-ALL patients with this fusion gene have been identified. Until now, fewer than 60 adult cases have been reported, among which over 40 cases have been diagnosed with T-ALL. The estimated incidence of the SET-CAN fusion gene in adult patients with T-ALL has been reported
Correspondence: Xiaoxue Wang; Hongcang Gu Email liang19569I@I63.com; gu_hongcang@hotmail.com 
to be $\sim 5 \%{ }^{6}$ On the cytogenetic level, it is unclear if the SET$C A N$ fusion is generated by a $\mathrm{t}(9 ; 9)(\mathrm{q} 34 ; \mathrm{q} 34)$ or an interstitial deletion at $9 \mathrm{q} 34$. The precise role of the SET-CAN fusion in hematopoietic cells and its contribution to leukemogenesis remains unknown. ${ }^{7}$ It is generally believed that the prognosis of such patients is poor and that these patients are insensitive to traditional chemotherapy and corticosteroids, so hematopoietic stem cell transplantation (HSCT) may improve the prognosis of such patients. ${ }^{8,9}$

Here, we report three patients carrying the SET-CAN fusion gene who were diagnosed with T-ALL, AML and myeloid sarcoma (MS), respectively. Furthermore, to the best of our knowledge, no cases of myeloid sarcoma carrying the SET-CAN fusion gene have been reported thus far. In the present study, the relevant literature regarding adult patients with the SET-CAN fusion gene was reviewed in order to provide a comprehensive profile of this rearrangement. This study was approved by the Ethics Committee of the First Affiliated Hospital of China Medical University. Written informed consent was obtained from all the three patients.

\section{Case Presentation Case I}

A 21-year-old male patient was admitted to our center in December 2019 due to lymphadenopathy with fever and fatigue. Ultrasonographic findings suggested splenomegaly and generalized lymphadenopathy on both sides of the diaphragm. Immunohistochemical staining for cervical lymph node biopsy displayed as diffused abnormal proliferative lymphoblastic cells with CD3(+) TdT(+) CD99(+) CD4(+) Ki67(70\%), while a few scattered cells were positive for MPO, CD117 and CD8. The patient was diagnosed with T-lymphoblastic lymphoma (LBL). The complete blood cell $(\mathrm{CBC})$ of the patient showed a white blood cell (WBC) count of $37.16 \times 10^{9} / \mathrm{L}$, a hemoglobin (HGB) level of $150 \mathrm{~g} / \mathrm{L}$ and a platelet level of $244 \times 10^{9} / \mathrm{L}$. Intriguingly, a bone marrow aspirate revealed hypercellularity with predominant blasts (Figure 1A), and flow cytometry showed the T-lymphoblasts (P3 group, 85.9\%) mainly expressed CD7, cCD3 and CD38; partially expressed CD5 and HLADR; and did not express CD33, CD117, CD34, CD19,
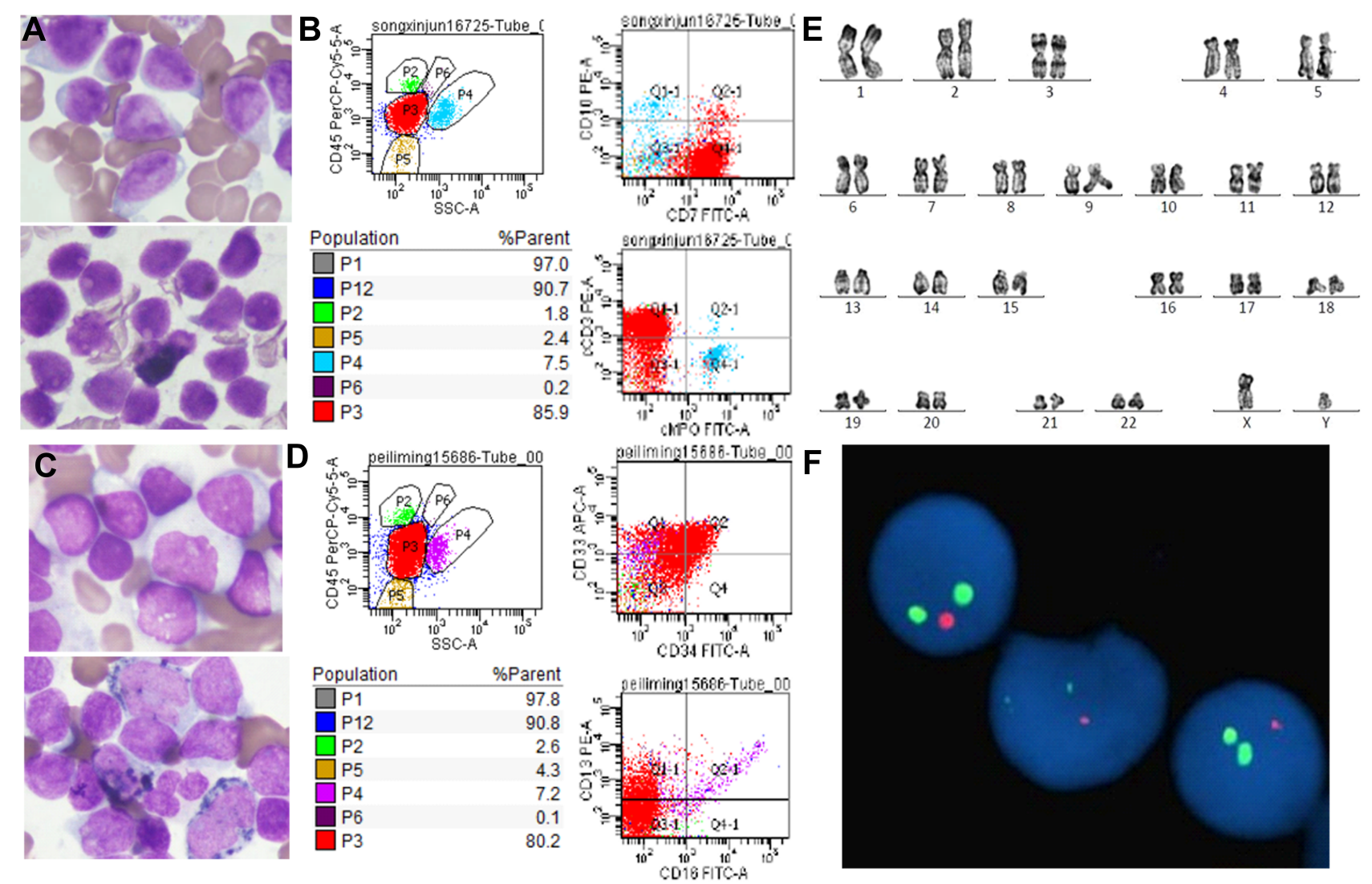

Figure I (A) Morphology of leukemic cells at diagnosis (original magnification, $\times 1000$ ) for case I. (B) Flow cytometry result for case I. (C) Morphology of leukemic cells at diagnosis (original magnification, $\times 1000$ ) for case 2. (D) Flow cytometry result for case 2. (E) Karyotype analysis showed normal result of case I. (F) Dual-color FISH analysis of case I with LSI BCR-ABLI dual-color, dual-fusion translocation probe showing a monoallelic loss of the $A B L$ gene. The $A B L$ gene $(9 q 34)$ was labeled as orange, and the $B C R$ gene (22q।I.2) was labeled as green. 
CD10, MPO, cCD79a, CD2, CD1a, CD15, CD13, CD56, TdT, CD123, CD25, CD99, CD4, CD8, CD3, TCRa/b, $\mathrm{TCRg} / \mathrm{d}$ and CD45RO, which indicated Pro-T-ALL (Figure 1B). Karyotyping analysis of a bone marrow (BM) sample illustrated that the patient had a 46,XY karyotype[20] (Figure 1E). In total, reverse transcriptase (RT)PCR covering 56 commonly detected fusion genes in leukemia (listed in Supplementary Table 1) was performed on the bone marrow sample. The SET-CAN fusion gene was detected. To determine whether the SET-CAN fusion identified in this case was derived from deletion of $9 q 34$, fluorescence in situ hybridization (FISH) analysis using the $B C R / A B L$ fusion probe covering this region was applied to the cultured bone marrow cells. The $A B L$ gene $(9 \mathrm{q} 34)$ was labeled as orange, and the $B C R$ gene (22q11.2) was labeled as green (Figure $1 \mathrm{~F}$ ). A total of 200 cells were analyzed, and $\sim 83 \%$ of the cells showed deletion of $A B L$ and two copies of $B C R$. The remaining cells showed a normal hybridization pattern. The FISH result was nuc ish $(\mathrm{BCR} \times 2),(\mathrm{ABL} \times 1)$ [166/200]. Then, next generation sequencing (NGS) was performed on 39 commonly mutated genes in ALL (listed in Supplementary Table 2), and we identified a missense mutation c. $1803 \mathrm{~A}>\mathrm{T}$ (p.Lys601Asn) in BRAF (NM_004333). Based on the clinical course and laboratory findings, the patient was finally diagnosed with T-LBL/ALL and subsequently received an induction chemotherapy VICP (vindesine $4 \mathrm{mg} / \mathrm{d}, \mathrm{d} 1,8,15,22$; idarubicin $8 \mathrm{mg} / \mathrm{d}$, d8-10; cyclophosphamide $1.2 \mathrm{~g} / \mathrm{d}, \mathrm{d} 8$; dexamethasone $15 \mathrm{mg} / \mathrm{d}$, d1-5, 11-14). Complete remission (CR) was achieved after the first cycle of chemotherapy. The patient will continue consolidation therapy and wait for allogeneic HSCT.

\section{Case 2}

A 24-year-old male presented with lymphadenopathy for half a month without fever and was admitted to our center in August 2019. The routine blood test showed a WBC count of $11.41 \times 10^{9} / \mathrm{L}$, a HGB level of $126 \mathrm{~g} / \mathrm{L}$ and a platelet level of $211 \times 10^{9} / \mathrm{L}$. CT findings suggested mediastinal and bilateral axillary lymphadenopathy as well as splenomegaly. The proportion of blasts in bone marrow was $81.2 \%$ (Figure 1C), and flow cytometry (Figure 1D) showed the blasts (P3 group, 80.2\%) mainly expressed CD7, CD33 and CD34; partially expressed CD11b, HLADR, CD123, CD64 and CD13; and did not express CD10, CD117, CD16, CD19, CD10, MPO, cCD3, cCD79a, CD14, CD3, CD15, CD4, CD8, CD2, CD25, CD9 and CD11c, which indicated AML. The karyotype result was normal. The SET-CAN fusion gene was detected in the bone marrow sample by RT-PCR. Then, NGS identified ainsertion mutation c.4834dupC (p.Leu1612fs) in the BCOR gene (NM_01123383) (38.51\%); a PHF6 (NM_001015877) mutation (85.21\%): c.746C $>\mathrm{T} \quad$ (p. Thr249Ile); and a CEBPA (NM_004364) mutation (6.1\%): c.857G $>$ A (p.Arg286Gln). The patient was diagnosed with AML-M5. A standard DA (Daunorubicin $120 \mathrm{mg} \times 3$ days, Cytarabine $200 \mathrm{mg} \times 7$ days) was given for two cycles; then, Cytarabine $3.5 \mathrm{~g} \mathrm{Q} 12 \mathrm{~h} \times 3$ days was given for five cycles. CR was achieved. The patient declined HSCT and has been alive for 8 months.

\section{Case 3}

A 32-year-old female presented with a mediastinal mass for 2 months with no fever and was admitted to our center in October 2018. The CBC of the patient showed a WBC count of $4.15 \times 10^{9} / \mathrm{L}$, an HGB level of $111 \mathrm{~g} / \mathrm{L}$ and a platelet level of $128 \times 10^{9} / \mathrm{L}$. Ultrasonographic findings suggested generalized lymphadenopathy. The result of Lung CT+ enhancement showed left hilar and mediastinal space occupying lesions, $5.6 \times 4.0 \mathrm{~cm}$ in diameter, with left upper lobe obstructive changes, pericardial effusion and left pleural effusion (Figure 2A). The PET-CT showed soft tissue shadow in the mediastinum and chest wall, wrapping mediastinal vessels, with SUVmax $=10.1$, which were considered to be malignant lesions. The left pleura was thickened with increased metabolism, and the metabolism of the left neck and upper and lower clavicle lymph nodes was also increased (Figure 2B). Immunohistochemical staining of the mediastinal mass biopsy (Figure 2C) displayed diffused abnormal proliferative cells with $\mathrm{CD} 7(+) \mathrm{Pax} 8(+)$ CD33(+)CD43(+)CD99(+) $\quad$ CD4(+) $\quad$ LMO-2(+) $\quad \mathrm{Ki67}$ $(85 \%)$, while a few scattered cells were positive for CD117, Pax5 and CD8. Other staining results showed CD1a(-), CD5(-), CD20(-), CD3(-), CK(-), CD19(-), CD21(-), CD163(-), EMA(-), P63(-), CD68(-), MPO (-), CD34(-), TdT(-), CAM5.2(-), TTF1(-), ALK(-), CD30(-), CD10(-), CD56(-), CgA(-), GB(-), SYN(-), TIA1(-) and EBER(-). The patient was diagnosed with myeloid sarcoma (MS). The results of bone puncture, bone marrow biopsy and bone marrow flow cytometry were all negative. The karyotype result was normal. The SET-CAN fusion gene was detected. Pericardial effusion was exudate. The Rivalta test was positive, and the cell count was $4481 \times$ $10^{6} / \mathrm{L}$. After the initial chemotherapy with an HIA regimen (Idarubicin $20 \mathrm{mg} \times 2$ days, cytarabine $100 \mathrm{mg} \mathrm{m}^{2} \times 7$ days, homoharringtonine $2 \mathrm{mg} \times 4$ days), the mediastinal mass was significantly reduced. However, the follow-up treatment did 

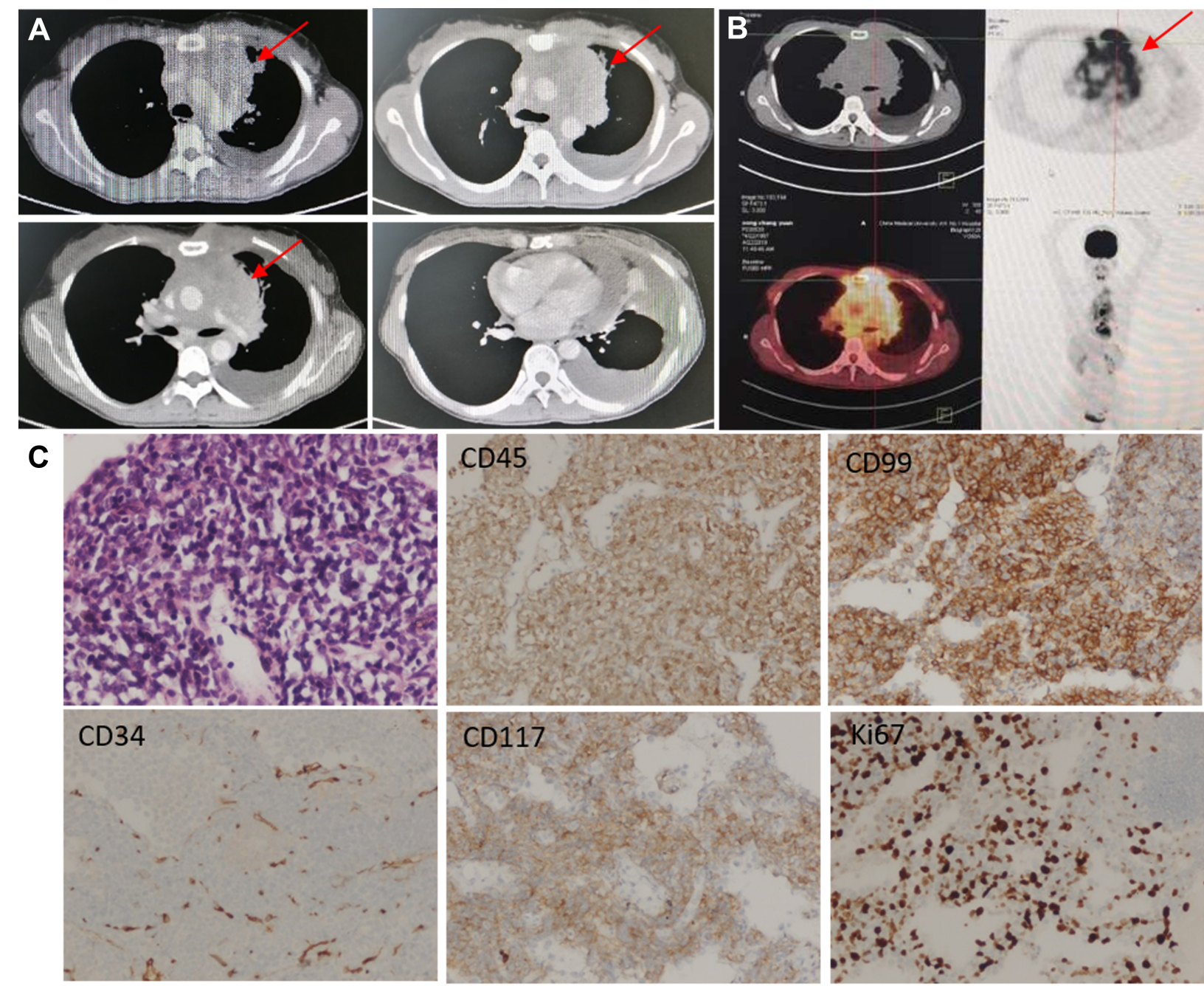

D

SET

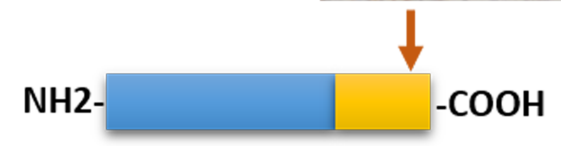

\section{Acidic domain}

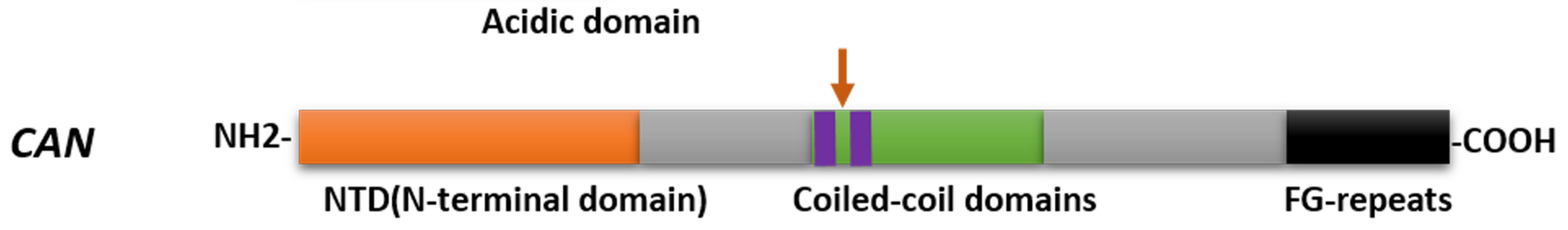

SET-CAN NH2-

$\mathrm{COOH}$

Figure 2 (A) The result of Lung CT+ enhancement for case 3.The tumor is marked by arrow. (B) The result of PET-CT for case 3. The tumor is marked by arrow. (C) Immunohistochemical staining of the mediastinal mass biopsy for case 3. (D) Schematic representation of SET, CAN, and SET-CAN proteins. Fusion breakpoints are indicated with vertical arrowhead.

not further alleviate the disease, and the negative effect of myelosuppression was particularly prominent, with a rapidly increased pericardial effusion. At last, the patient discontinued treatment due to intestinal infection.

\section{Discussion}

Both the SET and CAN genes are located at chromosome $9 \mathrm{q} 34$. The SET protein is a potent endogenous inhibitor of protein phosphatase $2 \mathrm{~A}(\mathrm{PP} 2 \mathrm{~A})$. It is overexpressed in 
numerous cancer types. ${ }^{10,11}$ The SET protein has multiple functions, being involved in, for example, apoptosis, the cell cycle and nucleosome assembly. ${ }^{12}$ CAN/NUP214 is a type of nucleoporin, which is the main component of the nuclear pore complex and plays a role in nuclear protein import, mRNA export and cell cycle progression. ${ }^{13}$ The SET-NUP214 fusion protein consists of almost the whole SET protein fused to the C-terminus of NUP214 (Figure 2D).

LBL/ALL is an aggressive malignant proliferative disease of the hematopoietic system. It is characterized by uncontrolled proliferation of T-lineage progenitor cells with a 5 -year-overall survival of $\sim 48 \%{ }^{14}$ The clinical features include presentation with hyperleukocytosis and extramedullary infiltration of the lymph nodes and other organs. This form accounts for $25 \%$ of total adult ALL cases. ${ }^{15}$ Most patients with T-ALL have a high tumor load, with rapid disease progression and a high risk of disease recurrence. Until now, just over 40 adult T-ALL patients with the SET-CAN fusion gene have been reported. Relevant articles with detailed information on patients have been collected in Table 1. Here, we collected eight studies, including 35 adult T-ALL patients with the SET-CAN fusion. ${ }^{6,9,16-21}$ Another two studies also mentioned patients with the SET-CAN fusion gene but were not included because they lacked the detailed information. ${ }^{22,23}$ Most of the reported T-ALL cases with the SET-CAN fusion gene occurred in young and middle-aged men. Flow cytometry analysis showed that patients not only had the differentiation antigen of $\mathrm{T}$ lymphocytes, but also expressed many myeloid antigens, suggesting that the tumor cells of these patients may be in the early stage of $\mathrm{T}$ lymphocyte development. The deletion or translocation of the small segment on chromosome 9 is difficult to detect using conventional cytogenetic methods, and the aberration needs to be further confirmed by FISH or array. More than one-third of the patients described in the literature had normal karyotype results. Complex karyotypes and other abnormal karyotypes have also been reported. However, del(9q34) can be detected in all patients who received FISH or array tests. Most of the patients presented with the SET exon7-NUP214 exon18 (S7N18 type) fusion transcript (13/14). Only one patient presented with the SET exon7-NUP214 exon17 (S7N17 type) fusion transcript (1/14). Mutation of the NOTCH1 gene as well as the PHF6 gene has also been identified in several patients.

It is reported that patients with a SET-CAN fusion have poor prognosis and are not sensitive to chemotherapy, especially to high dose glucocorticoids. It is suggested that HSCT should be carried out as early as possible after remission. Yang, et $\mathrm{al}^{9}$ reported three patients with T-ALL harboring the SET-CAN fusion gene, all of whom were refractory to high dose glucocorticoid-based chemotherapy. The authors sorted CD $34^{+}$Lin- cells from one patient as primary T-ALL cells and found that these cells were insensitive to dexamethasone. Additionally, SET-CAN mediated the loss of regulation of histone $\mathrm{H} 3$ acetylation, which might be a potential mechanism of glucocorticoid resistance. Furthermore, CLAG chemotherapy in combination with asparaginase might be a potential treatment option for adult SET-CAN ${ }^{+}$T-ALL patients. The SET-CAN fusion gene is also considered to be a contributor to the poor responsiveness of SET-CAN-harboring leukemic cells to glucocorticoids. In one study, the SET-CAN fusion protein did not interact with the glucocorticoid receptor, was constitutively coprecipitated with glucocorticoid response elements and suppressed glucocorticoid receptor transcriptional activity and histone acetylation. ${ }^{24}$ In our case report, patient 1 showed a normal karyotype result accompanied with del(9q34) confirmed by FISH. A missense mutation of $B R A F$, which has not been previously reported in patients harboring the SETCAN fusion gene, was identified by NGS. The mutant BRAF protein continuously activates the Ras/BRAF signaling pathway, which is essential for tumor growth, proliferation, invasion and metastasis. ${ }^{25}$

While the majority of the adult patients experienced T-ALL, other subtypes of acute leukemia with the SETCAN fusion gene are summarized in Table 2. Here, we collected seven studies ${ }^{4,5,7,8,26-28}$ including nine adult patients with the SET-CAN fusion gene. Three patients were diagnosed with AUL, four patients were diagnosed with AML and the other two were diagnosed with B-ALL. Another study conducted by Choi et $\mathrm{al}^{23}$ also mentioned two cases of AML, but was not included in our table because it lacked detailed information. According to the literature, only one B-ALL patient was female, while all the patients with AML and AUL were male. The median age was 36.5 years (19-46 years). In our report, case 2 was diagnosed with AML-M5. A mutation in PHF6 was identified in this patient, which has also been mentioned in other patients with the SET-CAN fusion gene. ${ }^{18}$ PHF6, located in the nucleolus, is an X-linked tumor suppressor gene which functions in transcriptional regulation. PHF6 mutations can be found in $15 \%$ of AML patients and is associated with poor prognosis. ${ }^{29}$ Additionally, BCOR gene mutations have been found in $8-10 \%$ of AML 


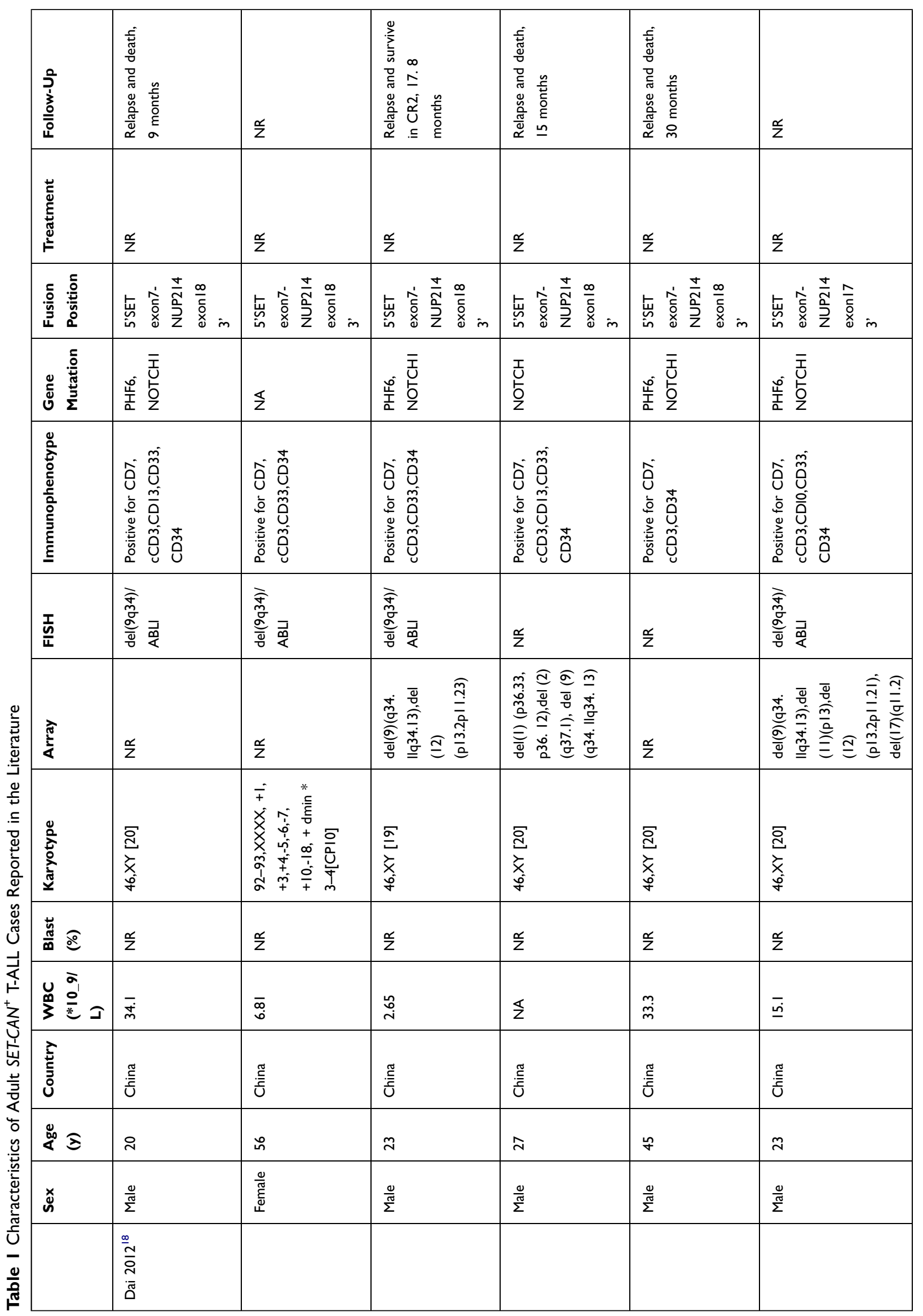




\begin{tabular}{|c|c|c|c|c|c|c|c|}
\hline 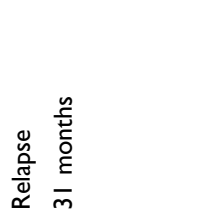 & 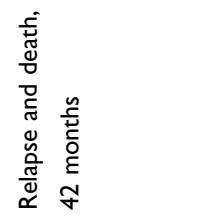 & 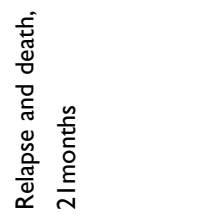 & 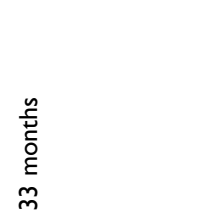 & 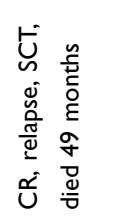 & 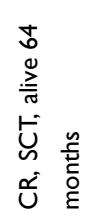 & 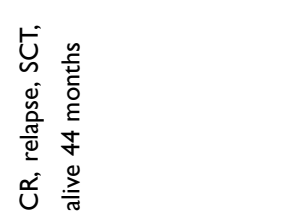 & 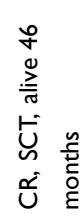 \\
\hline$\frac{\alpha}{z}$ & $\stackrel{\alpha}{Z}$ & $\frac{\alpha}{z}$ & $\stackrel{\alpha}{Z}$ & 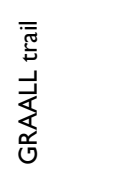 & 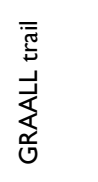 & 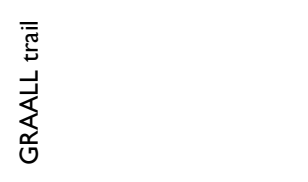 & 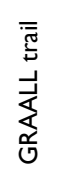 \\
\hline 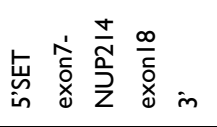 & 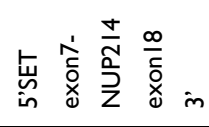 & 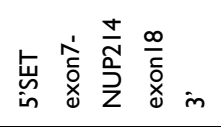 & 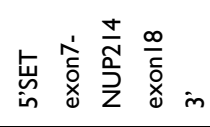 & $\stackrel{\alpha}{z}$ & $\stackrel{\alpha}{z}$ & $\stackrel{\alpha}{Z}$ & $\stackrel{\alpha}{z}$ \\
\hline$\frac{\alpha}{z}$ & $\frac{\alpha}{Z}$ & $\stackrel{\alpha}{z}$ & $\frac{\alpha}{z}$ & $\frac{\alpha}{z}$ & $\stackrel{\alpha}{z}$ & $\frac{\alpha}{z}$ & $\frac{\alpha}{z}$ \\
\hline 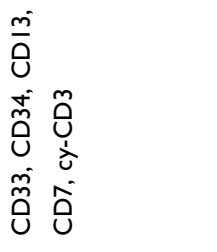 & 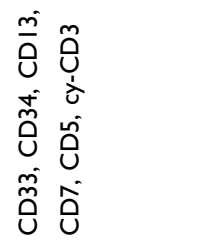 & 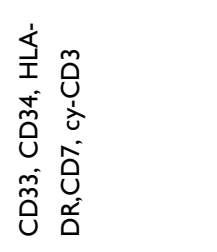 & 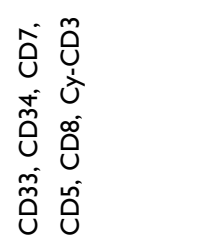 & 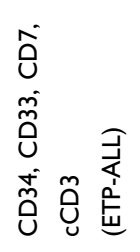 & 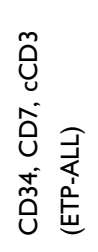 & 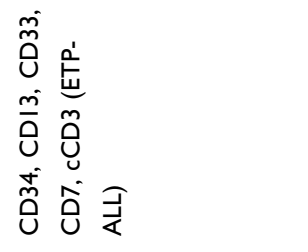 & 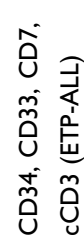 \\
\hline 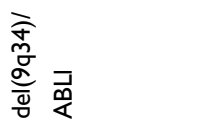 & 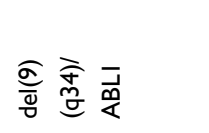 & 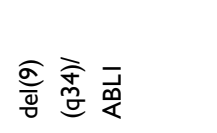 & 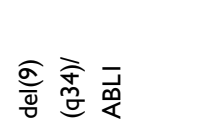 & $\stackrel{\alpha}{z}$ & $\stackrel{\alpha}{z}$ & $\stackrel{\mathscr{c}}{z}$ & $\stackrel{\alpha}{Z}$ \\
\hline 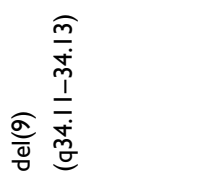 & $\stackrel{\alpha}{Z}$ & $\frac{\alpha}{z}$ & $\stackrel{\alpha}{z}$ & $\stackrel{\alpha}{z}$ & 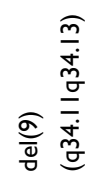 & 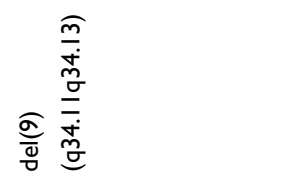 & $\frac{\alpha}{z}$ \\
\hline 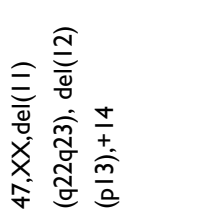 & 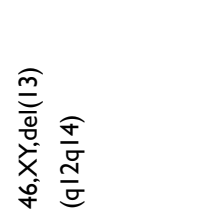 & 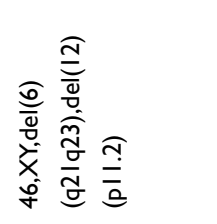 & 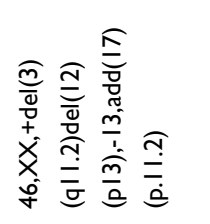 & 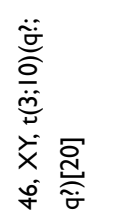 & 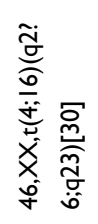 & 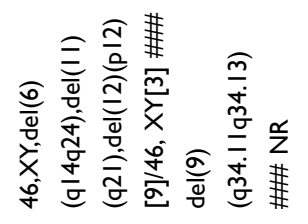 & 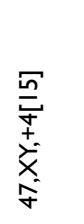 \\
\hline$\infty$ & ఓ & $\delta$ & œ & $\stackrel{\alpha}{z}$ & $\stackrel{\alpha}{z}$ & $\stackrel{\alpha}{z}$ & $\stackrel{\alpha}{z}$ \\
\hline 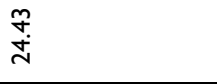 & $\begin{array}{l}\stackrel{+}{\Delta} \\
\underline{\infty}\end{array}$ & 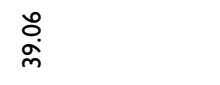 & î̀ & ¿্ं & $\stackrel{0}{\infty}$ & $\bar{o}$ & $\underset{\stackrel{\infty}{\infty}}{\underline{\sigma}}$ \\
\hline $\begin{array}{l}\stackrel{\Xi}{0} \\
\stackrel{0}{0}\end{array}$ & $\begin{array}{l}\stackrel{\Xi}{0} \\
\stackrel{0}{0}\end{array}$ & 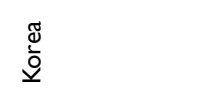 & 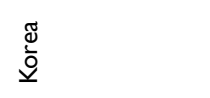 & 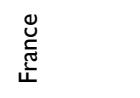 & 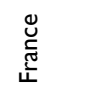 & 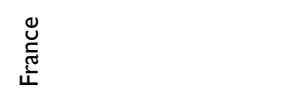 & 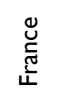 \\
\hline in & $\tilde{m}$ & $\approx$ & 오 & $\stackrel{+}{m}$ & $\hat{m}$ & ๙े & $\bar{\sigma}$ \\
\hline $\begin{array}{l}\frac{0}{\tilde{\Phi}} \\
\frac{\tilde{E}}{\omega}\end{array}$ & $\frac{\frac{\omega}{\tilde{N}}}{\Sigma}$ & $\frac{\frac{\omega}{\tilde{N}}}{\Sigma}$ & 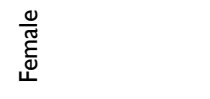 & $\frac{\frac{\omega}{\pi}}{\Sigma}$ & 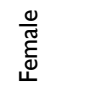 & $\frac{\frac{\omega}{\tilde{N}}}{\Sigma}$ & $\frac{\frac{\omega}{\pi}}{\Sigma}$ \\
\hline 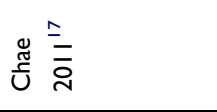 & & & & • & & & \\
\hline
\end{tabular}




\begin{tabular}{|c|c|c|c|c|c|}
\hline 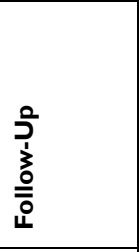 & 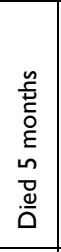 & 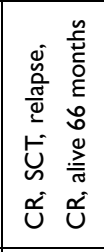 & 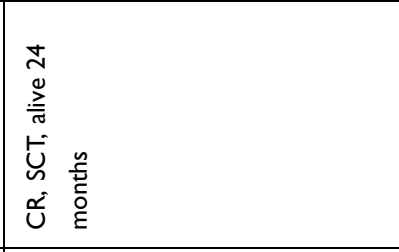 & 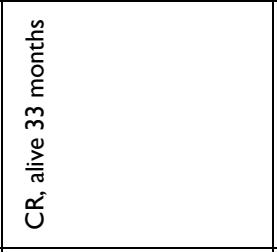 & 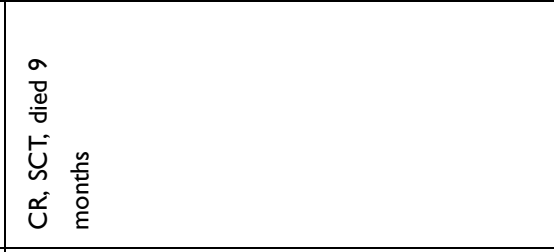 \\
\hline 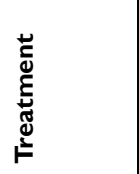 & 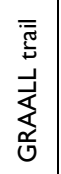 & 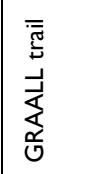 & 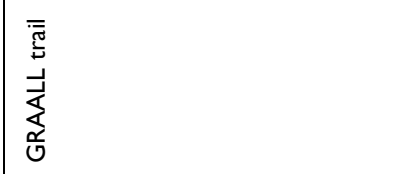 & 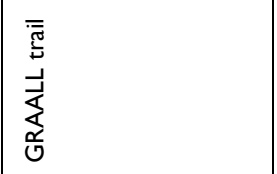 & 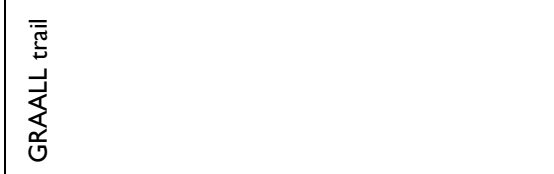 \\
\hline 亳耪 & $\frac{\infty}{z}$ & $\frac{\mathscr{L}}{z}$ & $\frac{\alpha}{z}$ & $\stackrel{\mathscr{Z}}{z}$ & $\stackrel{\mathscr{\alpha}}{z}$ \\
\hline 总 & $\frac{o}{z}$ & ž & $\frac{\alpha}{z}$ & $\stackrel{\alpha}{z}$ & $\frac{\alpha}{z}$ \\
\hline 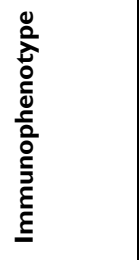 & 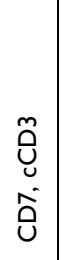 & $\begin{array}{l}0 \\
\tilde{u} \\
\tilde{0} \\
\hat{U}\end{array}$ & 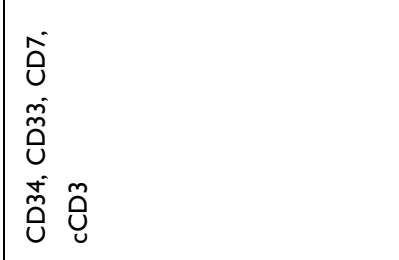 & $\begin{array}{l}0 \hat{u} \\
\tilde{u} \\
\tilde{u}\end{array}$ & 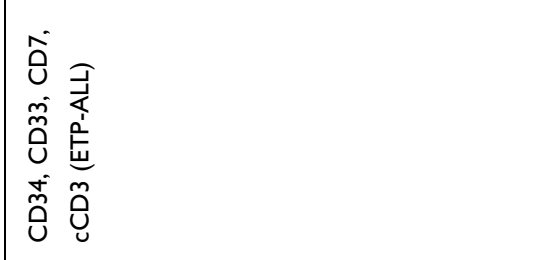 \\
\hline$\frac{I}{\frac{I}{L}}$ & $\frac{\infty}{z}$ & $\frac{\alpha}{z}$ & $\frac{\alpha}{z}$ & $\stackrel{\alpha}{z}$ & $\stackrel{\alpha}{z}$ \\
\hline 产 & $\frac{\mathscr{a}}{z}$ & $\frac{x}{z}$ & $\stackrel{\infty}{z}$ & $\stackrel{\alpha}{z}$ & $\stackrel{\alpha}{z}$ \\
\hline 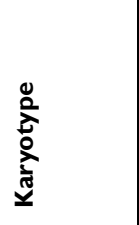 & 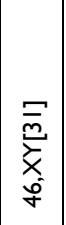 & 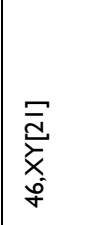 & 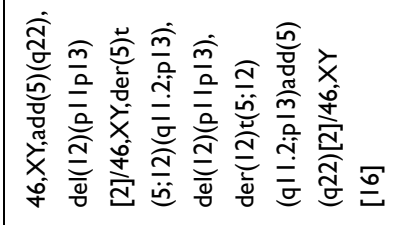 & 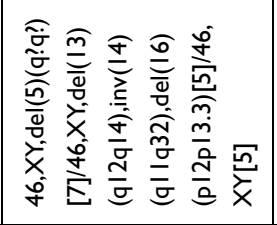 & 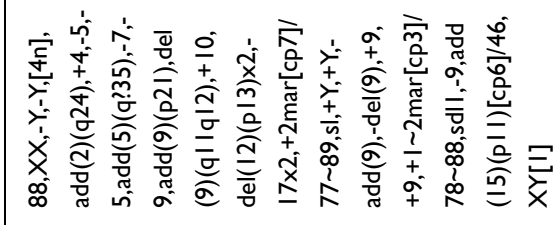 \\
\hline$\frac{\overrightarrow{\mathrm{a}}}{\mathrm{\omega}}$ ఠ & $\frac{\alpha}{z}$ & $\stackrel{\infty}{z}$ & $\frac{\infty}{z}$ & $\stackrel{\infty}{z}$ & $\frac{\alpha}{z}$ \\
\hline 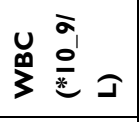 & $\begin{array}{l}+ \\
\dot{d} \\
\dot{0}\end{array}$ & $\stackrel{\leftrightarrow}{\dot{d}}$ & $\frac{\stackrel{\infty}{0}}{\underline{\underline{\infty}}}$ & 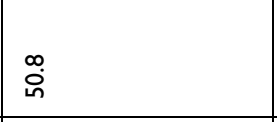 & $\underset{\sim}{\infty}$ \\
\hline نे & 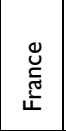 & 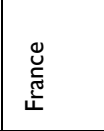 & 崖 & 总 & 总 \\
\hline 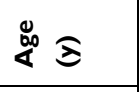 & $\approx$ & \% & $\stackrel{m}{m}$ & ஜ & $\underset{m}{\infty}$ \\
\hline ڤ̊̀ & $\frac{\frac{\rho}{\Sigma}}{\Sigma}$ & $\frac{\frac{0}{\Sigma}}{\Sigma}$ & $\frac{\frac{0}{\tilde{\omega}}}{\Sigma}$ & $\frac{\frac{\omega}{\pi}}{\Sigma}$ & $\frac{\frac{0}{\pi}}{\Sigma}$ \\
\hline
\end{tabular}




\begin{tabular}{|c|c|c|c|c|}
\hline 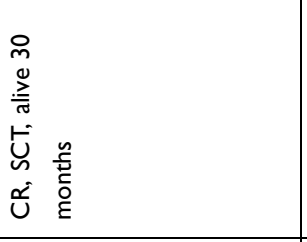 & 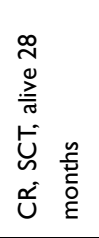 & 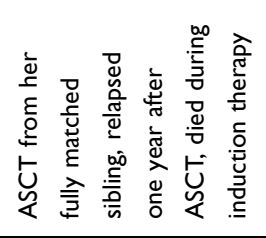 & 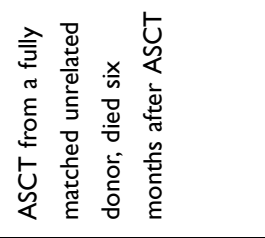 & 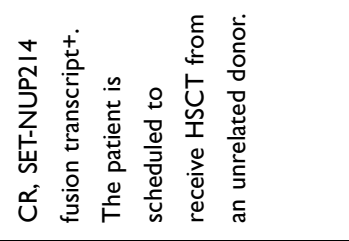 \\
\hline 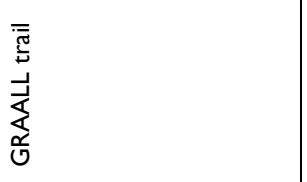 & 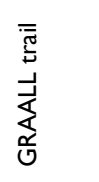 & 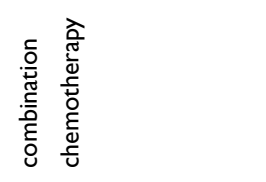 & 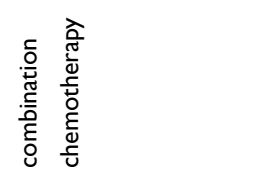 & 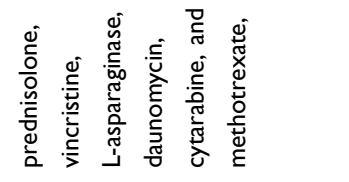 \\
\hline$\stackrel{\alpha}{Z}$ & $\frac{\alpha}{Z}$ & 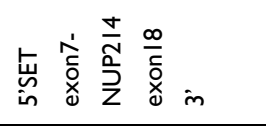 & 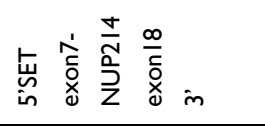 & 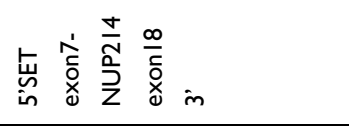 \\
\hline$\frac{\alpha}{z}$ & $\frac{\alpha}{z}$ & $\frac{\alpha}{Z}$ & $\frac{\alpha}{Z}$ & $\frac{\alpha}{z}$ \\
\hline 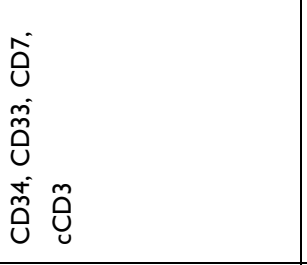 & $\begin{array}{l}\tilde{0} \\
\hat{u} \\
\hat{0}\end{array}$ & 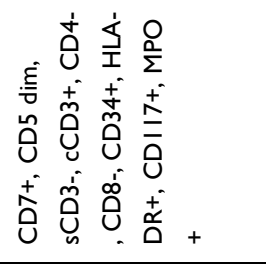 & 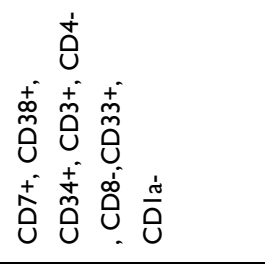 & 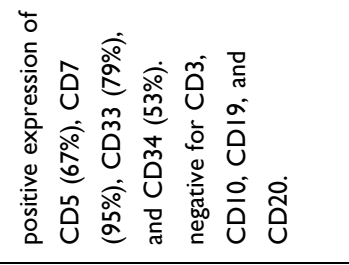 \\
\hline$\stackrel{\alpha}{Z}$ & $\frac{\alpha}{z}$ & $\frac{\alpha}{z}$ & $\frac{\alpha}{z}$ & $\frac{\alpha}{z}$ \\
\hline$\stackrel{\alpha}{z}$ & $\stackrel{\alpha}{z}$ & 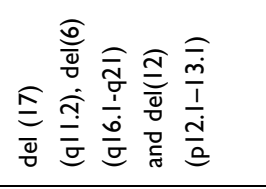 & 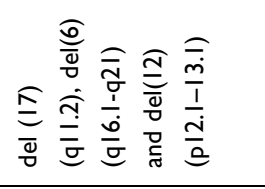 & $\stackrel{\mathscr{c}}{z}$ \\
\hline 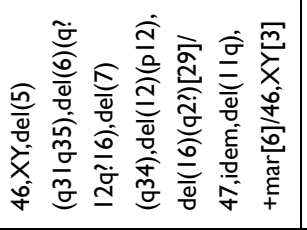 & 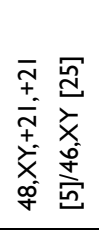 & $\stackrel{\alpha}{z}$ & 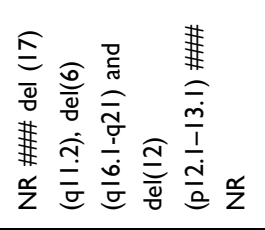 & 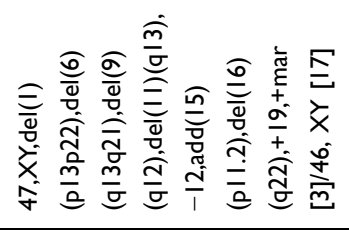 \\
\hline$\frac{o x}{z}$ & 号 & or & 号 & $\begin{array}{l}\text { Un } \\
\text { in }\end{array}$ \\
\hline$\stackrel{\infty}{\underset{\sigma}{\sigma}}$ & oे & $\frac{\alpha}{z}$ & $\frac{\alpha}{z}$ & 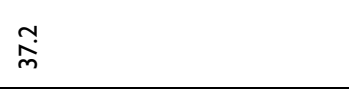 \\
\hline 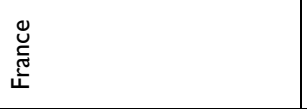 & 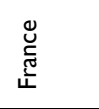 & 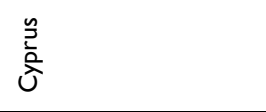 & $\begin{array}{l}\stackrel{n}{\underline{2}} \\
\overline{\hat{v}}\end{array}$ & 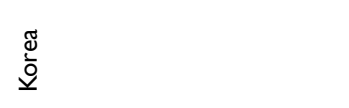 \\
\hline$\stackrel{\infty}{\sim}$ & ㅇ & $\stackrel{\infty}{+}$ & $\stackrel{\wp}{q}$ & $\stackrel{\infty}{\sim}$ \\
\hline \multirow[t]{2}{*}{$\frac{\frac{0}{\pi}}{\Sigma}$} & $\frac{\frac{\omega}{\pi}}{\Sigma}$ & $\begin{array}{l}\frac{0}{\pi} \\
\stackrel{\widetilde{E}}{\mathscr{N}} \\
\stackrel{L}{L}\end{array}$ & $\frac{\frac{\omega}{\tilde{\omega}}}{\frac{\pi}{\Sigma}}$ & $\frac{\frac{\omega}{\tilde{\omega}}}{\Sigma}$ \\
\hline & & 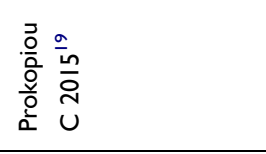 & & 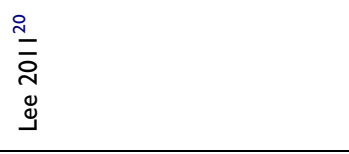 \\
\hline
\end{tabular}




\begin{tabular}{|c|c|c|}
\hline 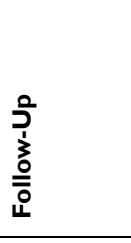 & 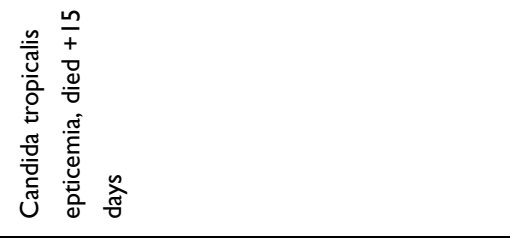 & 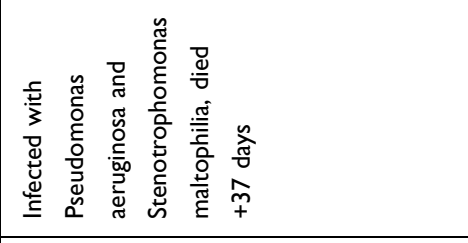 \\
\hline 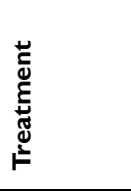 & 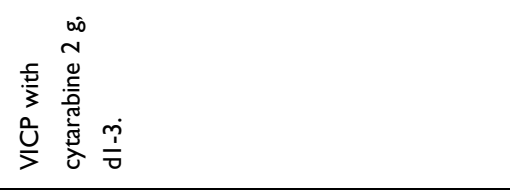 & 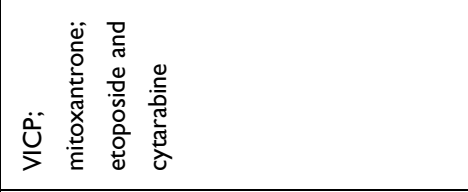 \\
\hline 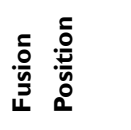 & $\frac{\infty}{z}$ & $\frac{\alpha}{z}$ \\
\hline 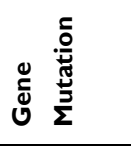 & $\frac{\infty}{z}$ & $\frac{\infty}{z}$ \\
\hline 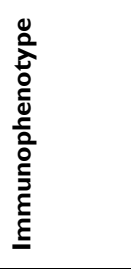 & 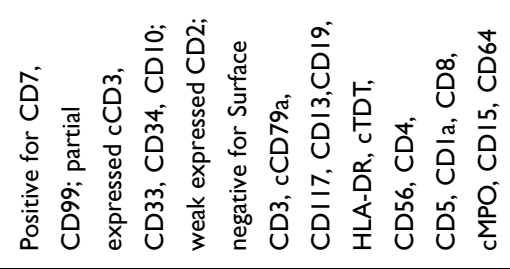 & 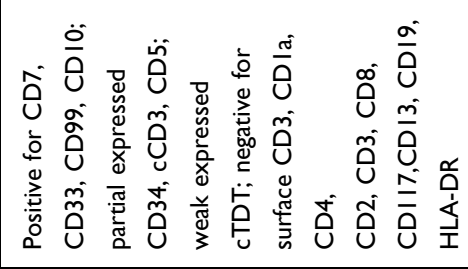 \\
\hline$\frac{\mathrm{I}}{\frac{\mathrm{D}}{\mathrm{U}}}$ & $\frac{\alpha}{z}$ & $\underline{\tilde{z}}$ \\
\hline 高 & $\frac{\alpha}{z}$ & $\frac{\alpha}{z}$ \\
\hline 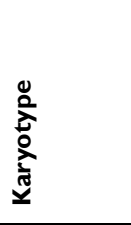 & 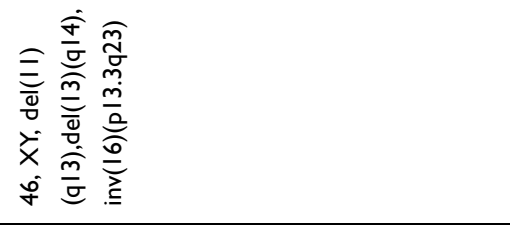 & $\begin{array}{l}\overline{\bar{g}} \\
\frac{\bar{z}}{2} \\
\frac{\bar{g}}{z}\end{array}$ \\
\hline$\frac{\breve{\mathrm{g}}}{\mathrm{\Phi}}$ ฮ & aे & $\begin{array}{c}\hat{\infty} \\
\infty\end{array}$ \\
\hline د & 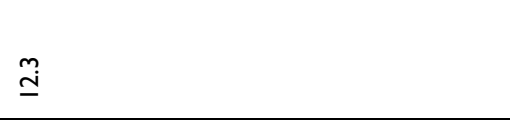 & $\overline{\bar{g}}$ \\
\hline 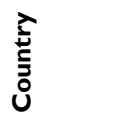 & 范 & 莺 \\
\hline 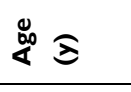 & ని & $\bar{n}$ \\
\hline ڤั & $\frac{\frac{0}{\Sigma}}{\Sigma}$ & $\frac{\frac{\varrho}{\Sigma}}{\Sigma}$ \\
\hline & 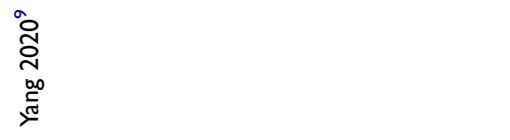 & \\
\hline
\end{tabular}




\begin{tabular}{|c|c|c|c|c|}
\hline 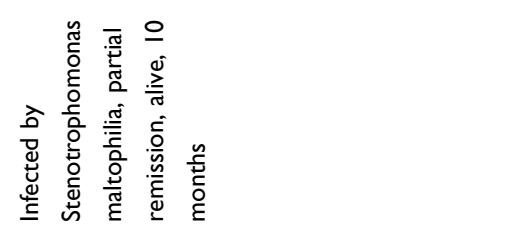 & $\stackrel{\alpha}{z}$ & 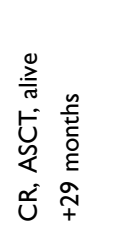 & 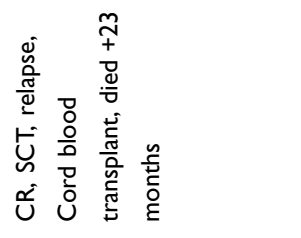 & 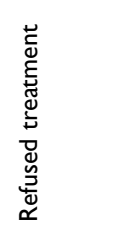 \\
\hline 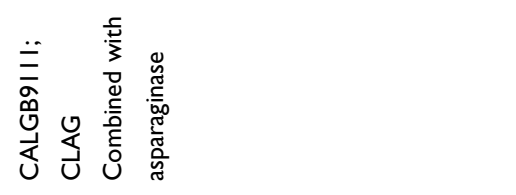 & $\stackrel{\alpha}{z}$ & $\stackrel{\alpha}{z}$ & $\stackrel{\alpha}{z}$ & $\stackrel{\alpha}{Z}$ \\
\hline$\frac{\alpha}{z}$ & 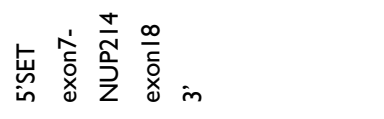 & $\stackrel{\alpha}{Z}$ & $\frac{\mathscr{O}}{Z}$ & $\stackrel{\alpha}{z}$ \\
\hline$\frac{\alpha}{Z}$ & $\stackrel{\alpha}{Z}$ & $\begin{array}{l}\text { I } \\
\text { o } \\
\text { Z - }\end{array}$ & $\begin{array}{l}\text { I } \\
\text { o } \\
\text { Z - }\end{array}$ & 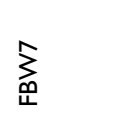 \\
\hline 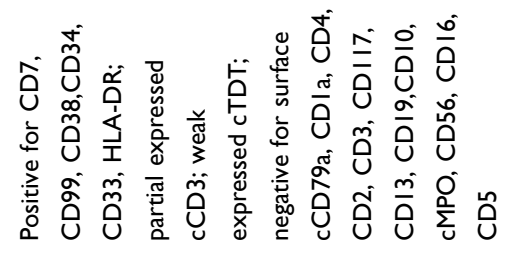 & 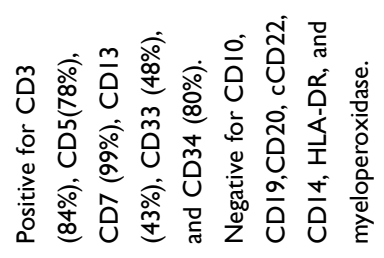 & tr & $\stackrel{b}{\frac{d}{2}}$ & 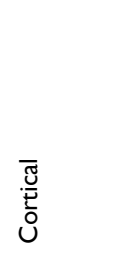 \\
\hline$\stackrel{\alpha}{z}$ & 产嵩 & 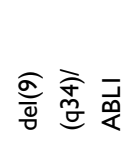 & 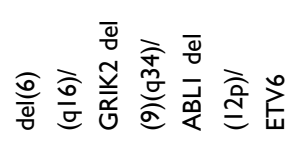 & 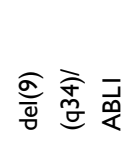 \\
\hline$\frac{\alpha}{z}$ & 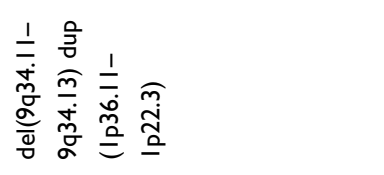 & $\stackrel{\alpha}{Z}$ & $\frac{o}{Z}$ & $\stackrel{o}{Z}$ \\
\hline 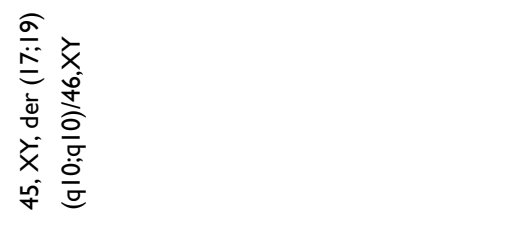 & 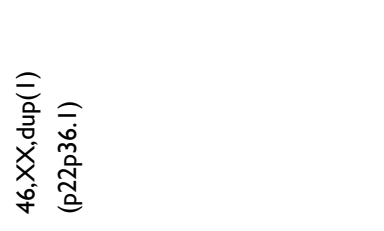 & 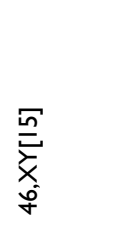 & 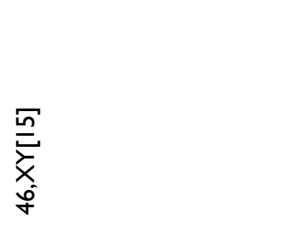 & 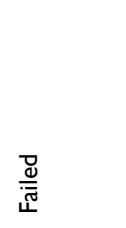 \\
\hline 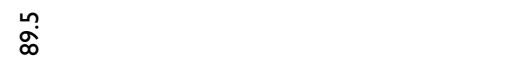 & $\stackrel{\llcorner}{\infty}$ & $\stackrel{\alpha}{z}$ & $\frac{\alpha}{z}$ & $\stackrel{\alpha}{z}$ \\
\hline$\stackrel{\stackrel{n}{m}}{\underline{m}}$ & :̊ & $\dot{\sim}$ & $\underset{\sim}{\infty}$ & $\stackrel{\alpha}{Z}$ \\
\hline . & 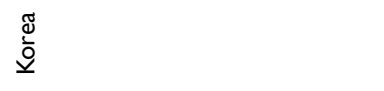 & 츨 & 츨 & 츨 \\
\hline$\hat{m}$ & $\mathscr{q}$ & $\stackrel{\infty}{m}$ & $\underline{a}$ & $\hat{f}$ \\
\hline$\frac{\frac{0}{\pi}}{\Sigma}$ & $\begin{array}{l}\frac{0}{\tilde{E}} \\
\frac{\tilde{E}}{\mathscr{L}}\end{array}$ & $\frac{\frac{\omega}{N}}{\Sigma}$ & $\frac{\frac{\omega}{\pi}}{\Sigma}$ & $\frac{\frac{\omega}{\pi}}{\Sigma}$ \\
\hline & 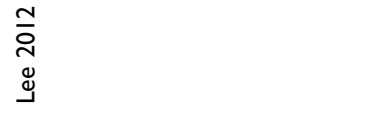 & $\begin{array}{l}\circ \\
\frac{0}{0} \\
\frac{0}{0}\end{array}$ & & \\
\hline
\end{tabular}




\begin{tabular}{|c|c|c|c|c|}
\hline 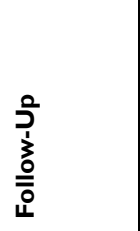 & 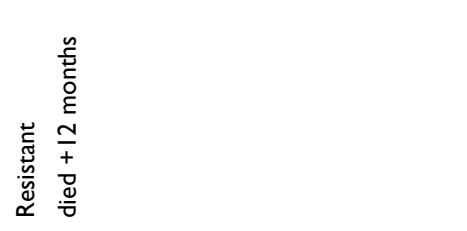 & 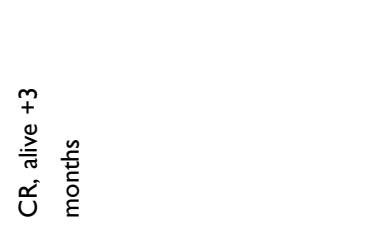 & 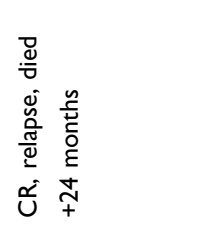 & 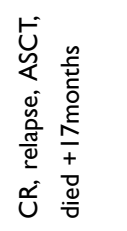 \\
\hline 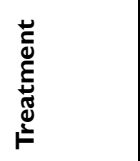 & $\frac{o}{Z}$ & $\frac{\alpha}{Z}$ & $\frac{\mathscr{N}}{Z}$ & $\frac{\mathscr{O}}{Z}$ \\
\hline$\frac{c}{\stackrel{0}{\underline{t}}}$ & $\stackrel{\alpha}{z}$ & $\stackrel{\alpha}{z}$ & $\stackrel{\alpha}{z}$ & $\frac{\mathscr{\alpha}}{z}$ \\
\hline 蓠 & $\begin{array}{l}\text { I } \\
\text { O } \\
\text { O }\end{array}$ & $\stackrel{\alpha}{z}$ & $\frac{\alpha}{z}$ & $\stackrel{\alpha}{Z}$ \\
\hline 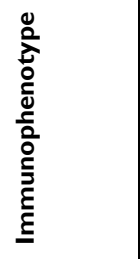 & $\stackrel{5}{\dot{d}}$ & $\frac{t_{0}}{2}$ & 它 & $\frac{\grave{g}}{\dot{2}}$ \\
\hline$\frac{I}{\frac{I}{L}}$ & 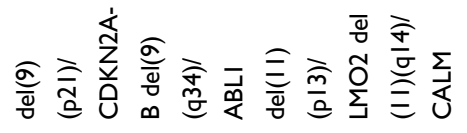 & 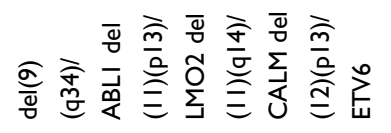 & 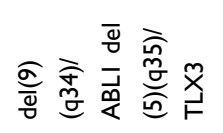 & 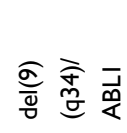 \\
\hline 䞤 & $\stackrel{\alpha}{Z}$ & 金 & 兑 & $\stackrel{o}{Z}$ \\
\hline 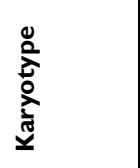 & 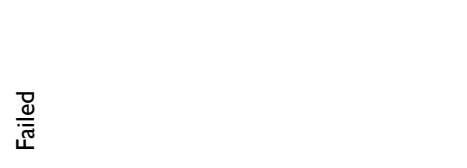 & 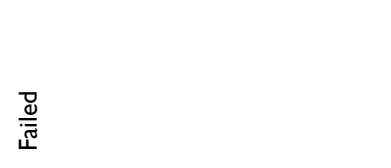 & 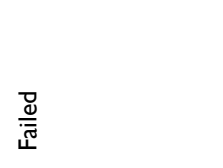 & 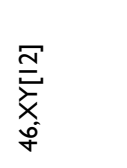 \\
\hline 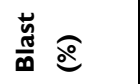 & $\frac{\alpha}{z}$ & $\stackrel{\alpha}{Z}$ & $\stackrel{\alpha}{z}$ & $\stackrel{o}{z}$ \\
\hline J & $\frac{\alpha}{z}$ & $\stackrel{\alpha}{z}$ & $\stackrel{o}{Z}$ & $\stackrel{\alpha}{z}$ \\
\hline uे & 츨 & 촡 & 촢 & 촢 \\
\hline 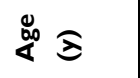 & $\hat{\sim}$ & $\underline{\underline{a}}$ & $\underline{\infty}$ & $\tilde{\sim}$ \\
\hline ڤ̊ & 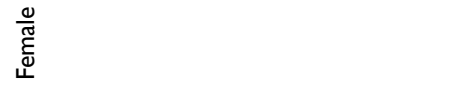 & $\frac{\frac{\omega}{\pi}}{\Sigma}$ & $\frac{\frac{\omega}{\pi}}{\Sigma}$ & $\frac{\frac{\omega}{\pi}}{\Sigma}$ \\
\hline
\end{tabular}




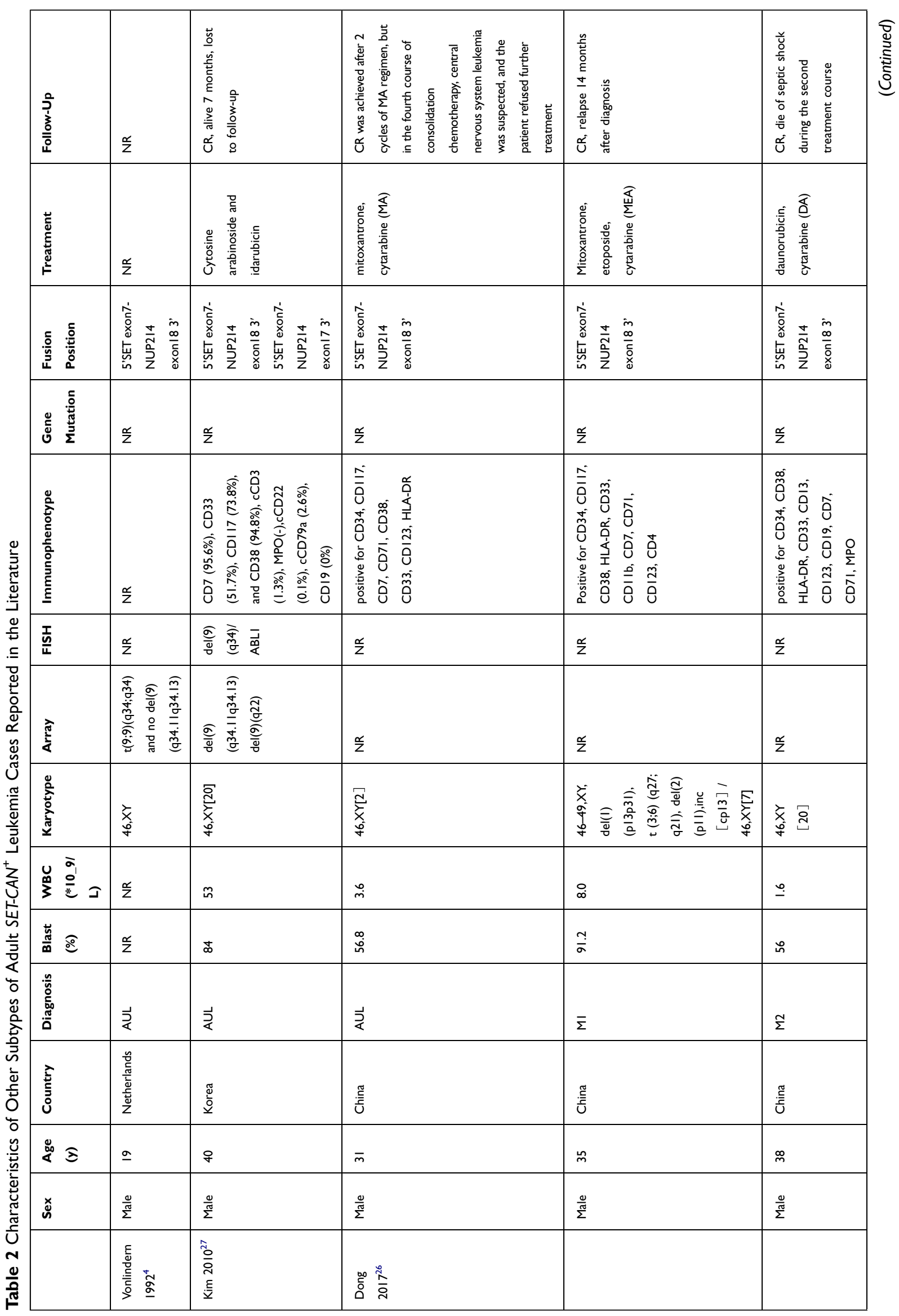




\begin{tabular}{|c|c|c|c|c|}
\hline 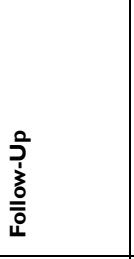 & 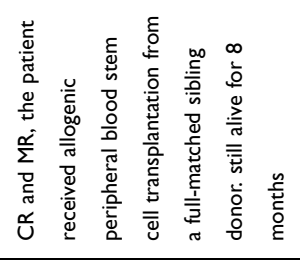 & 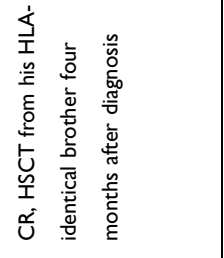 & 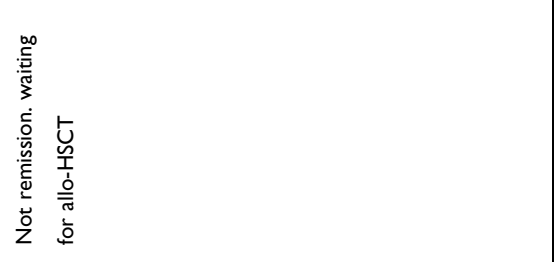 & $\frac{\alpha}{z}$ \\
\hline 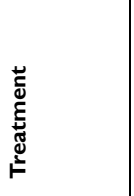 & 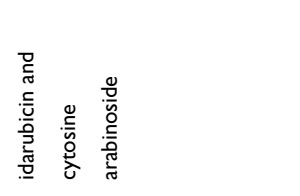 & 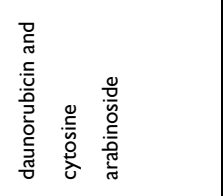 & 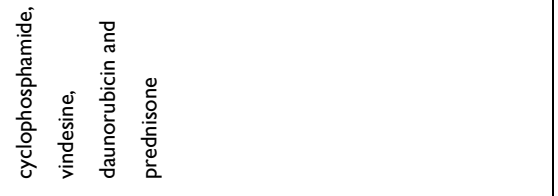 & $\frac{\alpha}{z}$ \\
\hline 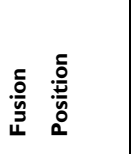 & 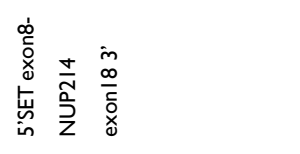 & 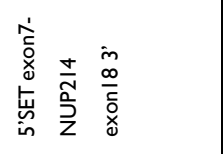 & 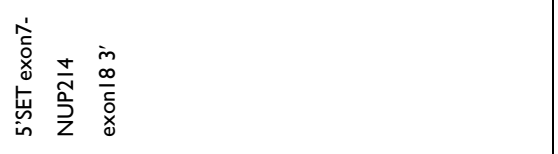 & 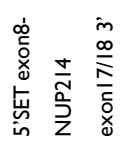 \\
\hline 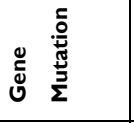 & $\frac{\alpha}{z}$ & $\stackrel{0}{\check{0}}$ & $\frac{\mathscr{N}}{\mathrm{Z}}$ & $\frac{\alpha}{z}$ \\
\hline 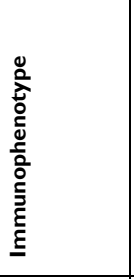 & 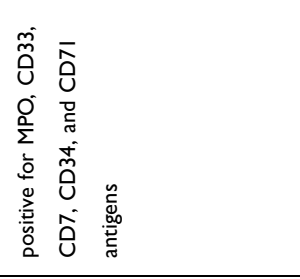 & 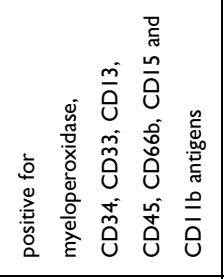 & 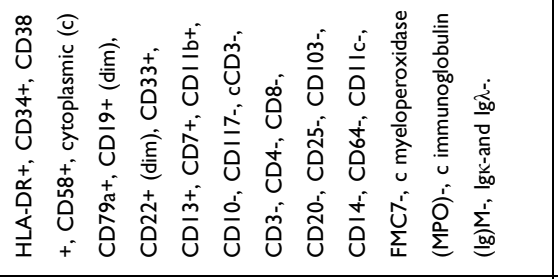 & $\stackrel{o}{z}$ \\
\hline$\frac{I}{\frac{I}{L}}$ & 产言 & 高高 & $\frac{\infty}{z}$ & 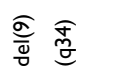 \\
\hline$\stackrel{\text { ते }}{\frac{\pi}{4}}$ & $\frac{\infty}{z}$ & 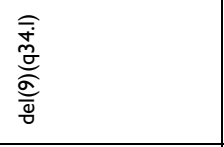 & $\frac{\alpha}{z}$ & 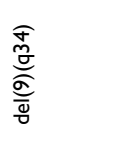 \\
\hline 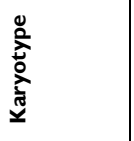 & 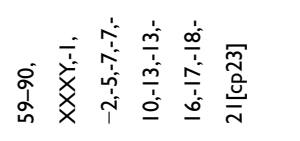 & $\begin{array}{l}\overline{\widetilde{g}} \\
\overline{\tilde{g}} \\
z\end{array}$ & 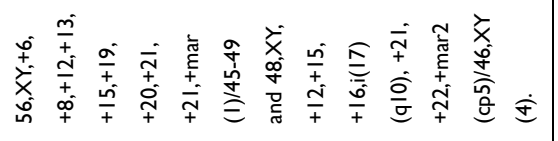 & $\begin{array}{l}\overline{\bar{g}} \\
\overline{\underline{g}} \\
\text { że }\end{array}$ \\
\hline 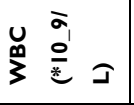 & $\overline{\check{I}}$ & q & $\overline{\bar{N}}$ & $\frac{\alpha}{z}$ \\
\hline 嵩 & $\infty$ & $\&$ & $\stackrel{\sim}{2}$ & $\frac{\alpha}{z}$ \\
\hline 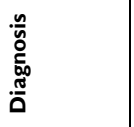 & $\bar{\Sigma}$ & $\sum_{\Sigma}^{ \pm}$ & 岁高 & 岇 \\
\hline نे & $\frac{\widetilde{g}}{0}$ & 츨 & 䗆 & 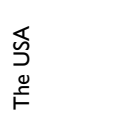 \\
\hline$\stackrel{\infty}{\star \infty}$ & f & $\stackrel{\sim}{m}$ & $\underline{a}$ & $\mathcal{F}$ \\
\hline ڤ̊̀ & $\frac{\frac{\omega}{\omega}}{\Sigma}$ & $\frac{\frac{\omega}{\tilde{\omega}}}{\Sigma}$ & $\frac{\frac{\varrho}{\omega}}{\Sigma}$ & $\begin{array}{l}\frac{\mathscr{0}}{\widetilde{E}} \\
\frac{\tilde{L}}{\mathscr{L}}\end{array}$ \\
\hline & 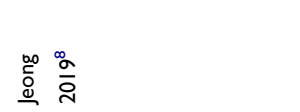 & 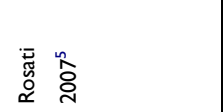 & 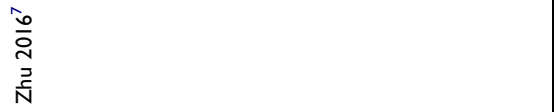 & 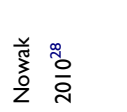 \\
\hline
\end{tabular}


cases and is usually associated with poor prognosis and secondary AML. ${ }^{30}$ The detected mutations in the present case have not been reported in the related literature.

Case 3 in our report was diagnosed with MS. This is a manifestation of extramedullary soft tissue masses which may develop as part of AML, myeloproliferative neoplasm, myelodysplastic syndrome or at relapse, especially in patients following allogeneic HSCT. ${ }^{31}$ Additionally, most of the literature about MS consists of case reports and small retrospective studies, and thus there is limited clinical knowledge of the cases and their presentation and management plans. ${ }^{32}$ Remarkably, this present case did not show any blast infiltration into the bone marrow, which is termed isolated or primary MS. Because high proportions of isolated MS patients may progress to AML, the recommended treatment regimen is conventional AML protocols. ${ }^{33}$

At present, research on the SET-CAN fusion gene mainly focuses on T-ALL. Although many studies have been performed on the SET-CAN fusion gene, the related clinical biological characteristics and the pathogenesis of leukemia are still unclear. Van Vlierberghe et $\mathrm{al}^{34}$ analyzed 92 patients with T-ALL. The SET-CAN fusion gene was identified in three patients and in the T-ALL cell line LOUCY. Further study revealed that the SET-CAN fusion gene inhibited the differentiation of T-cells by increasing the expression level of HOXA, thus promoting the occurrence of T-ALL. Similarly, another study conducted by Gorello et $\mathrm{al}^{6}$ showed that the SET-CAN fusion gene was identified in seven out of 152 patients with T-ALL. Subsequently, gene expression profiling identified a signature characterized by HOXA and NUP214 upregulation and $S E T$ downregulation. Quentmeier et al $^{11}$ performed RT-PCR-based screening of 141 leukemia/ lymphoma cell lines of T-, B- and myeloid cell origin to detect the SET-NUP214 fusion gene. That study only demonstrated the presence of the SET-NUP214 gene in the T-ALL cell line LOUCY and in the AML cell line MEGAL. Moreover, quantitative RT-PCR confirmed a positive correlation between SET-NUP214 and HOX gene expression in the cell line LOUCY when compared to six other T-ALL cell lines. Meanwhile, genomic sequencing localized the breakpoints of the SET gene to regions downstream of the stop codon and to NUP214 intron 17/18 in both the LOUCY and MEGAL cell lines.

As for the study of the SET-CAN fusion in the pathogenesis of leukemia, it has been reported that it may be related to aberrant intracellular localization of hCRM1, a nuclear export factor. Current research results indicated that SET and CAN were found in the nucleus and the nuclear envelope, respectively, whereas SET-CAN was primarily localized in the nucleus and interacts with hCRM1. Thus, the export of SET-CAN could be affected by hCRM1, which may lead to oncogenesis. ${ }^{35}$ Kandilci, et al ${ }^{36}$ verified that the SET-CAN fusion gene not only inhibits the differentiation of primitive progenitors but also committed myeloid cells (U937T) and therefore contribute to leukemogenesis. Subsequently, that same research group presented a transgenic mouse model that expresses the SET-CAN fusion gene in hematopoietic progenitor cells to further explore the role of SET-CAN in leukemogenesis. ${ }^{37}$ However, SET-CAN mice were not leukemia-prone and did not show shortening of disease latency after retroviral tagging. Surprisingly, SET-CAN mice developed spontaneous hyperplasia of the stomach mucosa, which indicated a role of SET-CAN in the proliferation of certain epithelial cells. A study conducted by Saito et $\mathrm{al}^{38}$ revealed that the SET-CAN fusion gene affected hematopoietic cell differentiation in a mouse model. Erythroid and megakaryocytic differentiation was impaired in SET-CAN transgenic mice.

\section{Conclusion}

In conclusion, the SET-CAN fusion gene was very rare in patients with leukemia, but was more prevalent in young men, most of whom are diagnosed with T-ALL. Conventional karyotype analysis was unable to detect this chromosomal abnormality, and the overall prognosis may be relatively poor. Allogeneic HSCT may improve the prognosis. However, there was a great heterogeneity between different patients. The clinical characteristics of SET-CAN positive patients and the pathogenesis of leukemia are not clear at present. The treatment efficacy and prognosis of patients may be also correlated with other genetic changes. More cases should be accumulated and summarized to better understand diseases related to this translocation.

\section{Ethical Statement}

Written informed consent to have the case details published was obtained from all the three patients, and the study was approved by Ethics Committee of the First Affiliated Hospital of China Medical University.

\section{Funding}

This work was supported by the National Natural Science Foundation of China (NSFC) (grant number: 81900153). 


\section{Disclosure}

The authors report no conflicts of interest for this work.

\section{References}

1. Shago M. Recurrent cytogenetic abnormalities in acute lymphoblastic leukemia. Methods Mol Biol. 2017;1541:257-278. doi:10.1007/9781-4939-6703-2 21

2. Song X, Peng Y, Wang X, et al. Incidence, survival, and risk factors for adults with acute myeloid leukemia not otherwise specified and acute myeloid leukemia with recurrent genetic abnormalities: analysis of the surveillance, epidemiology, and end results (SEER) database, 2001-2013. Acta Haematol. 2018;139(2):115-127. doi:10. $1159 / 000486228$

3. Raetz EA, Teachey DT. T-cell acute lymphoblastic leukemia. Hematology Am Soc Hematol Educ Program. 2016;2016 (1):580-588. doi:10.1182/asheducation-2016.1.580

4. von Lindern M, Breems D, van Baal S, Adriaansen H, Grosveld G. Characterization of the translocation breakpoint sequences of two DEK-CAN fusion genes present in $\mathrm{t}(6 ; 9)$ acute myeloid leukemia and a SET-CAN fusion gene found in a case of acute undifferentiated leukemia. Genes Chromosomes Cancer. 1992;5(3):227-234. doi:10. 1002/gcc.2870050309

5. Rosati R, La Starza R, Barba G, et al. Cryptic chromosome 9q34 deletion generates TAF-Ialpha/CAN and TAF-Ibeta/CAN fusion transcripts in acute myeloid leukemia. Haematologica. 2007;92 (2):232-235. doi:10.3324/haematol.10538

6. Gorello P, La Starza R, Varasano E, et al. Combined interphase fluorescence in situ hybridization elucidates the genetic heterogeneity of T-cell acute lymphoblastic leukemia in adults. Haematologica. 2010;95(1):79-86. doi:10.3324/haematol.2009.010413

7. Zhu HH, Zhao XS, Qin YZ, Lai YY, Jiang H. B-cell acute lymphoblastic leukemia associated with SET-NUP214 rearrangement: a case report and review of the literature. Oncol Lett. 2016;11 (4):2644-2650. doi:10.3892/ol.2016.4260

8. Jeong IH, An GD, Lim HH, et al. A rare case of acute myeloid leukemia with SET-NUP214 fusion and massive hyperdiploidy. Ann Lab Med. 2019;39(4):403-405. doi:10.3343/alm.2019.39.4.403

9. Yang Q, Qian H, Jin Z, et al. SET-CAN fusion gene as poor prognosis predictor in adult T-cell acute lymphoblastic leukemia. Leuk Lymphoma. 2020;61(1):217-220. doi:10.1080/10428194.2019.1660966

10. Christensen DJ, Chen Y, Oddo J, et al. SET oncoprotein overexpression in B-cell chronic lymphocytic leukemia and non-hodgkin lymphoma: a predictor of aggressive disease and a new treatment target. Blood. 2011;118(15):4150-4158. doi:10.1182/blood-2011-04-351072

11. Quentmeier H, Schneider B, Rohrs S, et al. SET-NUP214 fusion in acute myeloid leukemia- and T-cell acute lymphoblastic leukemia-derived cell lines. J Hematol Oncol. 2009;2(1):3. doi:10. $1186 / 1756-8722-2-3$

12. Cristobal I, Garcia-Orti L, Cirauqui C, et al. Overexpression of SET is a recurrent event associated with poor outcome and contributes to protein phosphatase $2 \mathrm{~A}$ inhibition in acute myeloid leukemia. Haematologica. 2012;97(4):543-550. doi:10.3324/ haematol.2011.050542

13. Bodoor K, Shaikh S, Enarson P, et al. Function and assembly of nuclear pore complex proteins. Biochem Cell Biol. 1999;77 (4):321-329. doi:10.1139/099-038

14. Marks DI, Rowntree C. Management of adults with T-cell lymphoblastic leukemia. Blood. 2017;129(9):1134-1142. doi:10.1182/blood2016-07-692608

15. Vadillo E, Dorantes-Acosta E, Pelayo R, Schnoor M. T cell acute lymphoblastic leukemia (T-ALL): new insights into the cellular origins and infiltration mechanisms common and unique among hematologic malignancies. Blood Rev. 2018;32(1):36-51. doi:10.1016/j. blre.2017.08.006
16. Ben Abdelali R, Roggy A, Leguay $\mathrm{T}$, et al. SET-NUP214 is a recurrent $\gamma \delta$ lineage-specific fusion transcript associated with corticosteroid/chemotherapy resistance in adult T-ALL. Blood. 2014;123 (12):1860-1863. doi:10.1182/blood-2013-08-521518

17. Chae H, Lim J, Kim M, et al. Phenotypic and genetic characterization of adult T-cell acute lymphoblastic leukemia with del(9)(q34); SET-NUP214 rearrangement. Ann Hematol. 2012;91(2):193-201. doi:10.1007/s00277-011-1289-x

18. Dai HP, Wang Q, Wu LL, et al. Expression of SET-NUP214 fusion gene in patients with T-cell acute lymphoblastic leukemia and its clinical significance. Zhongguo Shi Yan Xue Ye Xue Za Zhi. 2012;20 (5):1047-1051.

19. Prokopiou C, Koumas S, Neokleous N, Seimeni O, Barmpouti A. SET-NUP214 rearrangement in isolation is insufficient to induce leukemia: a single center experience. Leuk Lymphoma. 2016;57 (2):451-452. doi:10.3109/10428194.2015.1049169

20. Lee SG, Park TS, Cho SY, et al. T-cell acute lymphoblastic leukemia associated with complex karyotype and SET-NUP214 rearrangement: a case study and review of the literature. Ann Clin Lab Sci. 2011;41 (3):267-272.

21. Lee EY, Park TS, Kim MJ, et al. Detection of SET-NUP214 rearrangement using multiplex reverse transcriptase-polymerase chain reaction (RT-PCR) in acute leukemias: a case report and literature review on a Korean case series. Ann Hematol. 2012;91(7):1135-1138. doi:10.1007/s00277-011-1366-1

22. Chen X, Wang F, Zhang Y, et al. Retrospective analysis of 36 fusion genes in 2479 Chinese patients of de novo acute lymphoblastic leukemia. Leuk Res. 2018;72:99-104. doi:10.1016/j.leukres.2018. 08.009

23. Choi HJ, Kim HR, Shin MG, et al. Spectra of chromosomal aberrations in 325 leukemia patients and implications for the development of new molecular detection systems. J Korean Med Sci. 2011;26 (7):886-892. doi:10.3346/jkms.2011.26.7.886

24. Ichijo T, Chrousos GP, Kino T. Activated glucocorticoid receptor interacts with the INHAT component Set/TAF-Ibeta and releases it from a glucocorticoid-responsive gene promoter, relieving repression: implications for the pathogenesis of glucocorticoid resistance in acute undifferentiated leukemia with Set-Can translocation. Mol Cell Endocrinol. 2008;283(1-2):19-31. doi:10.1016/j.mce.2007. 10.014

25. Mirji G, Bhat J, Kode J, et al. Risk stratification of T-cell acute lymphoblastic leukemia patients based on gene expression, mutations and copy number variation. Leuk Res. 2016;45:33-39. doi:10.1016/j. leukres.2016.03.002

26. Dong XY, Li YL, Liu LS, et al. Clinical characteristics of four SET-NUP214 positive acute leukemia patients. Zhonghua Xue Ye Xue Za Zhi. 2017;38(12):1062-1065. doi:10.3760/cma.j.issn.02532727.2017.12.012

27. Kim J, Lee SG, Song J, et al. Molecular characterization of alternative SET-NUP214 fusion transcripts in a case of acute undifferentiated leukemia. Cancer Genet Cytogenet. 2010;201(2):73-80. doi:10.1016/j.cancergencyto.2010.05.010

28. Nowak NJ, Sait SN, Zeidan A, et al. Recurrent deletion of 9q34 in adult normal karyotype precursor B-cell acute lymphoblastic leukemia. Cancer Genet Cytogenet. 2010;199(1):15-20. doi:10.10 16/j.cancergencyto.2010.01.014

29. Papaemmanuil E, Gerstung M, Bullinger L, et al. Genomic classification and prognosis in acute myeloid leukemia. $N$ Engl $J$ Med. 2016;374(23):2209-2221. doi:10.1056/NEJMoa1516192

30. Ilyas AM, Ahmad S, Faheem M, et al. Next generation sequencing of acute myeloid leukemia: influencing prognosis. BMC Genomics. 2015;16(Suppl 1):S5. doi:10.1186/1471-2164-16-S1-S5

31. Vishnu P, Chuda RR, Hwang DG, Aboulafia DM. Isolated granulocytic sarcoma of the nasopharynx: a case report and review of the literature. Int Med Case Rep J. 2013;7:1-6. doi:10.2147/IMCRJ. S53612 
32. Magdy M, Abdel Karim N, Eldessouki I, Gaber O, Rahouma M, Ghareeb M. Myeloid Sarcoma. Oncol Res Treat. 2019;42 (4):224-229. doi:10.1159/000497210

33. Bakst RL, Tallman MS, Douer D, Yahalom J. How I treat extramedullary acute myeloid leukemia. Blood. 2011;118(14):3785-3793. doi:10.1182/blood-2011-04-347229

34. Van Vlierberghe $P$, van Grotel $M$, Tchinda $J$, et al. The recurren SET-NUP214 fusion as a new HOXA activation mechanism in pediatric T-cell acute lymphoblastic leukemia. Blood. 2008;111 (9):4668-4680. doi:10.1182/blood-2007-09-111872

35. Saito S, Miyaji-Yamaguchi M, Nagata K. Aberrant intracellular localization of SET-CAN fusion protein, associated with a leukemia, disorganizes nuclear export. Int $J$ Cancer. 2004;111(4):501-507. doi:10.1002/ijc. 20296
36. Kandilci A, Mientjes E, Grosveld G. Effects of SET and SET-CAN on the differentiation of the human promonocytic cell line U937. Leukemia. 2004;18(2):337-340. doi:10.1038/sj.leu.2403227

37. Ozbek U, Kandilci A, van Baal S, et al. SET-CAN, the product of the $t(9 ; 9)$ in acute undifferentiated leukemia, causes expansion of early hematopoietic progenitors and hyperproliferation of stomach mucosa in transgenic mice. Am J Pathol. 2007;171(2):654-666. doi:10.2353/ ajpath.2007.060934

38. Saito S, Nouno K, Shimizu R, Yamamoto M, Nagata K. Impairment of erythroid and megakaryocytic differentiation by a leukemia-associated and $\mathrm{t}(9 ; 9)$-derived fusion gene product, SET/TAF-Ibeta-CAN/Nup214. J Cell Physiol. 2008;214(2):322-333. doi:10.1002/jcp.21199

\section{Publish your work in this journal}

OncoTargets and Therapy is an international, peer-reviewed, open access journal focusing on the pathological basis of all cancers, potential targets for therapy and treatment protocols employed to improve the management of cancer patients. The journal also focuses on the impact of management programs and new therapeutic agents and protocols on patient perspectives such as quality of life, adherence and satisfaction. The manuscript management system is completely online and includes a very quick and fair peer-review system, which is all easy to use. Visit http://www.dovepress.com/ testimonials.php to read real quotes from published authors. 